\title{
AUXILIARY SENSORY CUES IMPROVE AUTOMATIC POSTURAL RESPONSES IN INDIVIDUALS WITH DIABETIC NEUROPATHY
}

\author{
Noel Rao $\mathrm{MD}^{1}$, Alexander S. Aruin $\mathrm{PhD}^{1,2}$ \\ ${ }^{1}$ Marianjoy Rehabilitation Hospital, Wheaton, IL, and ${ }^{2}$ University of Illinois at Chicago, IL
}

Corresponding author:

Alexander S. Aruin, Ph.D.

Department of Physical Therapy (MC 898)

University of Illinois at Chicago

1919 W. Taylor Street

Chicago, IL 60612

Tel: (312) 355-0904

Fax: (312) 996-4583

Email: aaruin@uic.edu

Word count: 3,527

Number of figures: 3

Number of tables: 1 


\begin{abstract}
Background. A loss of sensation in the lower limbs, observed in individuals with diabetes as well as the elderly, contributes to postural instability, altered gait patterns, increased risk of falling, and decreased quality of life.
\end{abstract}

Objective. To find out if somatosensory cues delivered to the intact tissues of the lower limbs above the ankle joints enhance the control of posture in individuals with peripheral neuropathy.

Methods. Twelve individuals with sensory neuropathy due to diabetes participated in static and dynamic balance tests with and without auxiliary sensory cues provided to the lower limbs without stabilizing the ankle joints. During the tests the subjects were required to stand on a fixed or moving computer-controlled platform with their eyes open or closed. Equilibrium scores and response latency were obtained.

Results. For all tests, equilibrium scores were significantly larger in experiments with auxiliary sensory cues in comparison to conditions without cues $(\mathrm{p}<0.05)$. Smaller latency scores were recorded in conditions with available auxiliary sensory information. The results indicate that auxiliary sensory cues provided to the intact tissues of the lower extremities could improve automatic postural responses in individuals with diabetic peripheral neuropathy.

Conclusions. The observed enhancement of automatic postural responses has clinical implications that aid in the understanding of postural control in individuals with peripheral neuropathy. The study outcome also provides a basis for future investigations on whether specially designed assistive means that provide auxiliary sensory cues could improve balance, mobility, and the performance of ADLs in individuals with peripheral neuropathy. 
Postural responses in individuals with peripheral neuropathy

Key words: Peripheral neuropathy; postural stability; auxiliary sensory cues 


\section{Postural responses in individuals with peripheral neuropathy}

\section{INTRODUCTION}

Approximately 12 million people have been diagnosed with diabetes in the US. This number is projected to increase to around 14.5 million by 2010, thus raising the cost associated with medical expenses and lost productivity to an estimated \$156 billion [1].

Individuals with diabetic neuropathy frequently experience somatosensory deficits such as loss of position, vibration, light touch sense, and sensory ataxia with the loss of ankle reflexes [2]. The loss of sensation secondary to diabetic distal sensory neuropathy has a markedly detrimental effect on postural stability [3]. Since ankle proprioception is a critical factor for postural stability $[4,5]$, peripheral neuropathy affects the way individuals with diabetes control their posture while standing. Thus, laboratory studies have demonstrated that individuals with peripheral neuropathy show a larger postural sway and sway velocity [6] and delayed postural responses to surface perturbations [7] than non-neuropathic diabetics.

In addition, the loss of sensation associated with diabetic neuropathy contributes to altered gait patterns, feelings of being less safe while standing and walking, increased risk of falling $[8,9]$ and decreased quality of life [10]. Literature data shows that among people ages 60 and over, individuals with diabetes had a 1.6 to 35-fold increase in falls compared to individuals without diabetes $[9,11]$. Diabetes also appears to increase the risk for fracture in the case of a fall [12].

Recent studies indicate that impaired limb sensation, linked to a large-fiber peripheral nerve dysfunction, is quite common in otherwise healthy people over the age of 65 and has become substantially more frequent with an increase in age [13]. In 


\section{Postural responses in individuals with peripheral neuropathy}

particular, age-related impairments in foot and ankle sensitivity have been shown to contribute to poor balance abilities and reduced mobility functions in the elderly [13, 14].

Several approaches were developed to address these problems. Among them are therapeutic shoes, inlays, and ankle foot orthoses (AFOs) that influence tactile and proprioceptive mechanisms resulting in improvements in balance and a reduced risk of falling [15]. It was reported that improvements in balance while using these devices are associated with the increased feedback from cutaneous receptors in the foot and ankle $[15,16]$. It was also shown that subsensory mechanical noise applied to the soles of the feet via vibrating insoles leads to reduced postural sway in healthy young and elderly individuals [17] and individuals with diabetic neuropathy [18].

A number of studies reported that the provision of additional somatosensory cues could attenuate postural sway in standing healthy subjects [19]. For example, contact of the index finger with a stationary surface was associated with the reduction of postural sway [19, 20]. It was suggested that the fingertip signal could specify the direction and velocity of the body motion and guide the execution of compensatory innervations to minimize body sway well before vestibular thresholds are exceeded [21]. Moreover, it was reported that sensory input to the hand and arm through a cane can reduce postural sway in individuals without a functioning vestibular system [22]. It has been also shown that the provision of an index finger touch on a stable surface could improve postural stability in individuals with peripheral neuropathy [23]. However, if this approach is implemented outside of the laboratory, it would require either using a cane [22] or touching walls. Neither approach is considered as a sound one. For example, using a cane makes one feel old [24] and nearly half of seniors who could benefit from using a cane or walker refuse to use one [25]. Conversely, 
providing sensory information to the lower extremities could potentially be an alternative approach helping individuals with peripheral neuropathy to better control their posture while walking. However, no literature data exists on whether the improvement of postural stability in individuals with peripheral neuropathy could be accomplished by providing additional sensory cues to the lower extremities.

Thus, the goal of the current study was to find out whether proprioceptive information delivered to the proximal parts of the lower legs could be used for the control of posture. We hypothesized that auxiliary sensory cues, that bypass the disrupted pathways in the lower legs, enhance the balance in individuals with diabetic neuropathy.

\section{METHODS}

\section{SUBJECTS}

Twenty three individuals with diabetic neuropathy referred by primary care physicians and neurologists to participate in the study were evaluated by a physician specialized in neurological rehabilitation. Twelve subjects were selected to participate in the

study based on satisfying the inclusion criteria. Inclusion criteria were: (1) clinically confirmed peripheral neuropathy with significant sensory loss and no other neurological or musculoskeletal disorders that could have affected their balance, such as ataxia due to central nervous system disorder or osteoarthritis of the knee, (2) ankle, knee and hip range of motion within normal limits, (3) strength at the toes, ankles, knees, and hips as determined by manual muscle tests of at least 3/5, (4) difficulty in balance maintenance and ambulation not associated with vestibular abnormalities, (5) ability to walk independently with or without 


\section{Postural responses in individuals with peripheral neuropathy}

any assistive devices, and (6) ability to stand unassisted for 5 min. There were nine men and three women; their mean age was $69.5 \pm 14.1$ years and the mean time from the disease onset was $14.4 \pm 6.2$ years. All subjects had clinically confirmed diabetes and sensory neuropathy based on testing for bilateral foot sensation using a Semmes-Weinstein aesthesiometry set (Research Designs Inc., Houston, TX) and vibration using $128 \mathrm{~Hz}$ tuning fork; they also had loss of muscle stretch reflexes. The Semmes-Weinstein monofilaments were applied to three dorsal and two plantar foot zones, five times at each site. Correct detection of touch provided by a filament in three out of five trials was considered to be that subject's sensory threshold [26]. The average score for the group of subjects was $4.71 \pm 0.9$, a score of more than 3.84 is associated with a diminished protective sensation. Sensitivity to vibration was also graded as normal, diminished, or absent by detection of the cessation of vibration applied with a 128 $\mathrm{Hz}$ tuning fork placed at the lateral malleolus and hallux [27]. Furthermore, we were able to corroborate the diagnosis in five subjects participating in the sensory nerve conduction study and found that all of them have an absence of sural sensory nerve action potentials. All subjects had numbness in their feet and all but one patient reported loss of balance while walking. The subjects' demographics are in presented in Table 1. The Semmes-Weinstein monofilaments and $128 \mathrm{~Hz}$ tuning fork technique were also used to asses the sensation above the ankle joint by applying monofilaments to the middle calf area and the tuning fork to the middle tibia. The tests revealed that all the subjects had normal sensation above the ankle joint (Table 1).

$<$ Table 1 is about here $>$ 


\section{Postural responses in individuals with peripheral neuropathy}

The study protocol was approved by the Marianjoy Rehabilitation Hospital Institutional Review Board. Prior to obtaining all participants’ written consent, the objectives and method of the study were explained.

\section{INSTRUMENTATION}

A Computerized Dynamic Posturography (CDP) technique (NeuroCom International Inc., OR) was used. The CDP includes the Sensory Organization (SOT) and Motor Control (MCT) tests. SOT evaluates the efficiency of the subject's proprioception in balance control using an equilibrium score (ES). ES is a measure of postural stability based on degrees of offset of the subject from the centered position in the anterior-posterior plane. The MCT assesses the efficacy of the patient's postural responses to platform translations of different magnitudes and directions. A measure of postural response to translations is based on calculating the time (latency) between the onset of the force platform translation and the start of a mechanical response initiated by the subject [28].

The use of CDP in the current study is justified by the following: First, the CDP allows quantifying of both the balance-stabilizing responses (during standing in an unperturbed stance with or without misleading visuals and/or lower leg proprioceptive information, SOT test) and balance-correcting responses (that involve support-surface

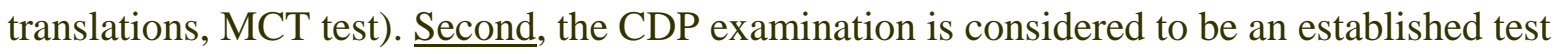
of postural stability [29] that is widely used for clinical and research purposes. The scientific literature describes multiple uses of the CDP tests in assessing balance in patients who have suffered from a stroke [30, 31], Parkinson disease [31], vestibular impairment [32], peripheral neuropathy [33, 34], elderly individuals [28, 35], children [36, 37], and in post 


\section{Postural responses in individuals with peripheral neuropathy}

space shuttle flights for astronauts [38]. Third, the analysis of the literature suggests that CDP is a reliable and valid technique [37]. For example, the literature states that the CDP "has fulfilled most criteria that would be required of a reliable and valid test of postural stability" [29]. It was also shown that healthy subjects retested on different days showed a minimal learning effect with CDP test repetitions [39]. In a recent study where CDP testing was used to explore the effects of galvanic stimulation, it was shown that SOT scores decreased during stimulation and returned to baseline thereafter, despite the retest experience of the subjects [40].

\section{PROTOCOL}

During the test session, subjects were asked to stand erect on the force platform with their hands at their sides. Their feet were shoulder width apart, with the medial malleoli over the placement strip imprinted on the platform. The instructions were to stand upright and relaxed throughout the test, with knees slightly bent and arms hanging loosely at the sides of the body. Prior to each testing condition subjects were instructed that during testing the force platform would move.

Sensory organization test. All subjects were exposed to 4 conditions that challenged their ability to integrate somatosensory, visual, and vestibular inputs in maintaining standing balance. Accordingly, in conditions 1 and 2, subjects stood on a fixed support surface with eyes open (SOT1) and closed (SOT2). The test conditions 4 and 5 involved standing on a sway-referenced support surface with eyes open (SOT4) and closed (SOT5).

Motor control test. The subjects were exposed to medium and large forward and backward perturbations of the platform. Each perturbation was applied three times within 


\section{Postural responses in individuals with peripheral neuropathy}

each trial. The duration of platform translations was 300 and 400 ms for the medium and large perturbations accordingly. The amplitude of translation was adjusted by computer software for differences in the subject's height. The sequence of testing was a medium and large backward translation of the platform followed by medium and large forward translation of the platform. The motor control test was administered with the eyes open. The response latency to perturbations that is defined as the time, expressed in milliseconds, between the onset of translation and the onset of the subject's active response to the support surface movement, was calculated.

Each subject participated in the SOT and MC testing sessions twice, first while standing in a regular erect posture (Fig 1A) that was not associated with any auxiliary sensory information and then with the device providing auxiliary sensory information without stabilizing the ankle joints (Fig. 1B). The device was designed as two Ankle Foot Orthoses, each of them consisting of the shank of the brace (1) connected with the foot bed (2) via a semi-rigid element (3). Velcro calf straps (4) were used to secure the shank of the brace to the leg. The device provided sensory information to the calf via the shank of the brace and to middle tibia via calf straps but at the same time did not provide any ankle stabilization as the connection of the shank of the brace with the foot bed was flexible. A licensed orthotic practitioner was involved in designing of the sensory cues only device.

To be sure that the shank of the brace connected with the foot bed via a semi-rigid element did not provide any ankle joint stabilization, we measured the horizontal force applied to the calf by that device in a pilot experiment. A miniature load cell, ELFS-B3-100N (Entran Devices, Fairfield, NJ), was taped to the inside of the shank of the brace at the level of the Velcro strap. The signal from the load cell was amplified (Coulbourn Instruments, 


\section{Postural responses in individuals with peripheral neuropathy}

Whitehall, PA) and collected at a sampling frequency of $100 \mathrm{~Hz}$ with 16-bit resolution using customized LabView software. The subject was standing vertical and then swayed backwards for several seconds, simulating sway-referenced CDP conditions. The force applied by the sensory cue only apparatus was about $0.9 \mathrm{~N}$. This value is below the force required to provide mechanical support but is associated with the provision of sensory cues $[19,41]$. Thus, the results of the pilot experiment confirmed that the device could provide sensory information while not restricting movements in the ankle joint. The order of tests was randomized.

The subjects wore the same standard shoes during all the tests and a safety harness that is required by the manufacturer of the Computerized Dynamic Posturography system. No feedback was given to subjects about their performance before the whole assessment was completed. One of the authors stood behind the subject throughout the duration of the tests to assist the subject in regaining balance if needed.

$<$ Fig 1 is about here $>$

\section{DATA ANALYSIS}

The SOT scores were obtained for each experimental condition to describe the efficiency of the subject's proprioception in balance control. The response latencies were calculated to quantify the subject's active response to the support surface movements. The independent variable was the availability of sensory information (Regular stance and standing with the device providing sensory cues). The dependent variables were SOT scores and latency of the postural responses. Separate repeated measures ANOVAs were performed on each of the two dependent variables. The LCD post hoc analysis was performed when 
needed. Significance levels for multiple comparisons were adjusted with the Bonferroni corrections.

\section{RESULTS}

The results of the calculation of the scores for the four testing conditions are presented in Fig. 1. In the first testing condition (SOT1), the subjects demonstrated scores of $92.37 \pm 0.55$ and $93.00 \pm 0.41$ without any devices and with the device providing auxiliary sensory information, respectively. In the second testing condition (SOT2), the scores were 85.29 \pm 0.97 and $86.80 \pm 0.88$, respectively. The scores for the fourth testing condition (SOT4) reached $76.75 \pm 4.90$ and $82.79 \pm 1.74$ for regular stance and with the device providing auxiliary sensory information, respectively. Finally, the scores for the SOT5 condition were 21.16 \pm 6.40 without any devices and $38.29 \pm 7.28$ with the device providing auxiliary sensory information. The improvement in the SOT scores due to the effect of the device providing auxiliary sensory information was statistically significant $(F 1,23=9.46, p<0.025)$. The effect of the testing conditions was also statistically significant $(F 3,69=93.61, \mathrm{p}<0.0001)$. Post hoc analysis revealed that the effect of the auxiliary sensory information was statistically significant in SOT2 (F1, $23=9.67, \mathrm{p}<0.005)$ and SOT5 (F1, $23=5.30, \mathrm{p}<$ $0.030)$ conditions.

\section{$<$ Fig 2 is about here $>$}

The composite latency scores that is an average of the individual scores for the two legs calculated for each experimental series are shown in Fig. 3. The mean composite latency score while standing with no auxiliary sensory cue device was $154.11 \pm 5.77 \mathrm{~ms}$. The latency 
decreased reaching $147.56 \pm 5.00 \mathrm{~ms}$ in the series with the sensory cues only device. The difference between the scores was not statistically significant $(p=0.20)$.

\section{$<$ Fig 3 is about here $>$}

\section{DISCUSSION}

Studies reported the importance of proprioceptive inflow to scale the postural adjustments [42]. Moreover, several reports suggested that the ability of humans to obtain early directionally sensitive proprioceptive information is not limited to finger touch. Thus, it was reported that postural sway is reduced when contact of the forehead, nose, or leg at nonsupportive force levels with a stable surface is provided [21]. Similarly, it was described that proprioceptive input from more proximal muscles, such as gluteus medius and paraspinals, could provide early directionally sensitive proprioceptive information [43]. It was also shown that ankle-foot orthoses (AFOs) improve automatic postural responses in individuals with peripheral neuropathy due to diabetes [33, 34]. However, as it was suggested in our previous study [34], improvement in automatic postural responses could be a result of auxiliary sensory cues or additional mechanical support that became available via the AFOs. The current study was designed to distinguish between the two possible sources of improvement of automatic postural responses by using the device that provided sensory cues without additional stabilization of the ankle joints.

There are two major findings of the study that support the study hypothesis that auxiliary sensory cues that bypass the disrupted pathways in the lower legs enhance the balance in individuals with diabetic neuropathy. 


\section{Postural responses in individuals with peripheral neuropathy}

First, improvement in the SOT scores were seen in all experimental conditions with the auxiliary sensory information compared to regular stance, a condition in which no additional sensory information was provided. The largest improvement of automatic postural reactions was seen in the two most difficult conditions in which either the somatosensory information was compromised through sway-referencing of the surface on which the subjects were standing (SOT4) or both the somatosensory and visual information was distorted through sway-referencing of the surface and the elimination of visual input (SOT5).

It is important to mention that while the effect of auxiliary sensory information was seen in all experimental conditions, the role of additional sensory cues was the largest in the SOT5 condition in which visual information was not available. When vision is not available, the CNS relies on information from two other systems, the vestibular and somatosensory systems [44]. There was no change in the utilization of vestibular information between the experimental conditions, as such we believe that a primary reason for the improvement in automatic postural responses seen in individuals with impaired proprioception was due to the availability auxiliary sensory cues.

Second, overall the subjects demonstrated a 4.7\% decrease in response latency when they were standing with the device delivering sensory information. This decrease in the time needed to respond to the sudden translation of the platform might reflect the fact that sensory information indeed was used in automatic postural responses. Why the time between the onset of translation and the onset of the subject's active response to the support surface movement was reduced only slightly? It is known that the loss of proprioception due to sensory neuropathy that usually develops over a long period of time, has a markedly detrimental effect on postural stability [3]. Since all the subjects have had a long history of 


\section{Postural responses in individuals with peripheral neuropathy}

diabetic distal polyneuropathy, it is possible that making available auxiliary sensory cues for a short period of time is not enough for the CNS to re-learn how to integrate this information. Indeed, it is known that if the CNS has to reweigh new sensory information, it might require substantial time to establish new means of integration of information needed for efficient postural control [45]. Given the fact that the subjects were exposed only to a limited number of body perturbations while using auxiliary sensory cues, additional training might be needed to enhance their ability to integrate sensory information for postural control.

It is necessary to point out that the experimental protocol included utilization of the harness system; thus, one could suggest that it provided additional support to the body and increased body stability that might have affected the outcome. We believe this was not the case, since the harness was attached loose and used as a means of protection against a possible fall. Moreover, the literature data showed that the use of a loose safety harness had no effects on body sway during quiet standing [46]. Nevertheless, the effect of the harness system as a source of sensory information still needs to be studied further.

Very few studies reported some effect of visual feedback during perturbations of a support platform [47] and exercise interventions on improving physical functioning and balance in individuals with peripheral neuropathy $[48,49]$. However, there is not much information available on the existence of other interventions aimed at reducing the functional consequences of peripheral neuropathy in order to enhance one’s mobility and quality of life [50]. In contrast, the results of the current study demonstrate that provision of auxiliary sensory information by bypassing the disrupted pathways in the lower legs could substitute for the lack of proprioceptive feedback from the lower extremities and as such to help individuals with peripheral neuropathy to generate faster and more sensitively scaled postural 


\section{Postural responses in individuals with peripheral neuropathy}

responses while standing. The findings also are consistent with previous studies of the effect of external vibrotactile biofeedback on stance [51] and gait [52]. Moreover, the observed improvements of automatic postural responses suggest that the device providing auxiliary sensory information could benefit the performance of other dynamic activities such as compensatory stepping in response to an unexpected platform perturbation and walking. If the device indeed acts as a "balance prosthesis" helping an individual with peripheral neuropathy to generate faster and more sensitively scaled postural responses during the gait cycle, its use could help such individuals to walk with less fear of falls and as such contribute to an improved quality of life.

The outcome of this study constitutes the first steps toward assessing the clinical significance of using auxiliary sensory cues to improve balance control in individuals with sensory deficits. The next step would be designing and evaluating advanced devices delivering auxiliary sensory cues to the calf area while still possessing the comfort such as for example, socks incorporating an "exoskeleton" that forms directly onto a lightweight and breathable fabric. Future studies are needed to determine whether devices providing auxiliary sensory cues are effective in enhancing performance of other dynamic balance activities (e.g, walking) and reducing the incidence of falls. This could be done running field-based monitoring and comprehensive prospective studies.

\section{CONCLUSION}

The results of the study revealed that automatic postural responses in individuals with diabetic peripheral neuropathy could be improved by using a device that allows sensory information bypassing the disrupted pathways in the lower legs. It also provides a basis for 
future investigations on whether specially designed assistive means that provide auxiliary sensory cues could improve balance and the performance of ADLs in individuals with peripheral neuropathy.

\section{ACKNOWLEDGEMENT}

This work was supported by the NIH grant HD51628. We would like to thank the patients for their exceptional cooperation, Dan Hasso, CPO for his help in designing AFOs, and Cheryl Carlson, PT for her help in testing.

\section{REFERENCES}

1. Hogan P, Dall T, Nikolov P. Economic costs of diabetes in the US in 2002. Diabetes Care. 2003;26:917-932.

2. Vinik AI, Holland MT, Le Beau JM, Liuzzi FJ, Stansberry KB, Colen LB. Diabetic neuropathies. Diabetes Care. 1992;15:1926-1975.

3. Simoneau GG, Ulbrecht JS, Derr JA, Becker MB, Cavanagh PR. Postural instability in patients with diabetic sensory neuropathy. Diabetes Care. 1994;17:1411-1421.

4. Teasdale N, Stelmach GE, Breunig A. Postural sway characteristics of the elderly under normal and altered visual and support surface conditions. J Gerontol. 1991;46:B238244.

5. Woollacott MH, Shumway-Cook A, Nashner LM. Aging and posture control: changes in sensory organization and muscular coordination. Int J Aging Hum Dev. 1986;23:97-114. 
6. Uccioli L, Giacomini PG, Monticone G, et al. Body sway in diabetic neuropathy. Diabetes Care. 1995;18:339-344.

7. Inglis JT, Horak FB, Shupert CL, Jones-Rycewicz C. The importance of somatosensory information in triggering and scaling automatic postural responses in humans. Exp Brain Res. 1994;101:159-164.

8. Cavanagh PR, Derr JA, Ulbrecht JS, Maser RE, Orchard TJ. Problems with gait and posture in neuropathic patients with insulin-dependent diabetes mellitus. Diabet Med. 1992;9:469-474.

9. Richardson JK, Hurvitz EA. Peripheral neuropathy: a true risk factor for falls. $J$ Gerontol A Biol Sci Med Sci. 1995;50:M211-215.

10. Benbow SJ, Wallymahmed ME, MacFarlane IA. Diabetic peripheral neuropathy and quality of life. Qjm. 1998;91:733-737.

11. Gregg EW, Beckles GL, Williamson DF, et al. Diabetes and physical disability among older U.S. adults. Diabetes Care. 2000;23:1272-1277.

12. Forsen L, Meyer HE, Midthjell K, Edna TH. Diabetes mellitus and the incidence of hip fracture: results from the Nord-Trondelag Health Survey. Diabetologia. 1999;42:920925.

13. Resnick HE, Vinik AI, Schwartz AV, et al. Independent effects of peripheral nerve dysfunction on lower-extremity physical function in old age: the Women's Health and Aging Study. Diabetes Care. 2000;23:1642-1647.

14. Bergin PS, Bronstein AM, Murray NM, Sancovic S, Zeppenfeld DK. Body sway and vibration perception thresholds in normal aging and in patients with polyneuropathy. $J$ Neurol Neurosurg Psychiatry. 1995;58:335-340. 
15. Hijmans JM, Geertzen JH, Dijkstra PU, Postema K. A systematic review of the effects of shoes and other ankle or foot appliances on balance in older people and people with peripheral nervous system disorders. Gait \& posture. 2007;25:316-323.

16. You SH, Granata KP, Bunker LK. Effects of circumferential ankle pressure on ankle proprioception, stiffness, and postural stability: a preliminary investigation. The Journal of orthopaedic and sports physical therapy. 2004;34:449-460.

17. Priplata AA, Niemi JB, Harry JD, Lipsitz LA, Collins JJ. Vibrating insoles and balance control in elderly people. Lancet. 2003;362:1123-1124.

18. Priplata AA, Patritti BL, Niemi JB, et al. Noise-enhanced balance control in patients with diabetes and patients with stroke. Annals of neurology. 2006;59:4-12.

19. Jeka JJ, Lackner JR. Fingertip contact influences human postural control. Exp Brain Res. 1994;100:495-502.

20. Clapp S, Wing AM. Light touch contribution to balance in normal bipedal stance. Experimental brain research Experimentelle Hirnforschung. 1999;125:521-524.

21. Lackner JR, Rabin E, DiZio P. Stabilization of posture by precision touch of the index finger with rigid and flexible filaments. Exp Brain Res. 2001;139:454-464.

22. Jeka JJ. Light touch contact as a balance aid. Phys Ther. 1997;77:476-487.

23. Dickstein R, Shupert CL, Horak FB. Fingertip touch improves postural stability in patients with peripheral neuropathy. Gait Posture. 2001;14:238-247.

24. O'Brian A. Most seniors shun canes: Survey. 2009 [cited 12/27/2009]; Available from:

http://www.leaderpost.com/health/seniors/Most+seniors+shun+canes+Survey/1426771/story. html 
25. O'Brian A. Seniors refuse help to avoid dangerous falls. The Vancouver Sun. 2009 March 24, 2009.

26. Holewski JJ, Stess RM, Graf PM, Grunfeld C. Aesthesiometry: quantification of cutaneous pressure sensation in diabetic peripheral neuropathy. J Rehabil Res Dev. 1988;25:1-10.

27. Horak FB, Dickstein R, Peterka RJ. Diabetic neuropathy and surface swayreferencing disrupt somatosensory information for postural stability in stance. Somatosens Mot Res. 2002;19:316-326.

28. Camicioli R, Panzer VP, Kaye J. Balance in the healthy elderly: posturography and clinical assessment. Arch Neurol. 1997;54:976-981.

29. Monsell EM, Furman JM, Herdman SJ, Konrad HR, Shepard NT. Computerized dynamic platform posturography. Otolaryngol Head Neck Surg. 1997;117:394-398.

30. Bonan IV, Colle FM, Guichard JP, et al. Reliance on visual information after stroke. Part I: Balance on dynamic posturography. Arch Phys Med Rehabil. 2004;85:268-273.

31. Toole T, Hirsch MA, Forkink A, Lehman DA, Maitland CG. The effects of a balance and strength training program on equilibrium in Parkinsonism: A preliminary study. NeuroRehabilitation. 2000;14:165-174.

32. Artuso A, Garozzo A, Contucci AM, Frenguelli A, Di Girolamo S. Role of dynamic posturography (Equitest) in the identification of feigned balance disturbances. Acta Otorhinolaryngol Ital. 2004;24:8-12.

33. Rao N, Aruin A. The Effect of Ankle-Foot Orthoses on Balance Impairment: SingleCase Study. Journal of Prosthetics and Orthotics. 1999;11:15-19 
34. Rao N, Aruin AS. Automatic postural responses in individuals with peripheral neuropathy and ankle-foot orthoses. Diabetes Res Clin Pract. 2006;74:48-56.

35. Wolfson L, Whipple R, Derby CA, et al. A dynamic posturography study of balance in healthy elderly. Neurology. 1992;42:2069-2075.

36. Casselbrant ML, Furman JM, Rubenstein E, Mandel EM. Effect of otitis media on the vestibular system in children. Ann Otol Rhinol Laryngol. 1995;104:620-624.

37. Gabriel LS, Mu K. Computerized platform posturography for children: test-retest reliability of the sensory test of the VSR System. Phys Occup Ther Pediatr. 2002;22:101117.

38. Paloski WH, Black FO, Metter EJ. Postflight balance control recovery in an elderly astronaut: a case report. Otol Neurotol. 2004;25:53-56.

39. Allum JH, Shepard NT. An overview of the clinical use of dynamic posturography in the differential diagnosis of balance disorders. J Vestib Res. 1999;9:223-252.

40. Macdougall HG, Moore ST, Curthoys IS, Black FO. Modeling postural instability with Galvanic vestibular stimulation. Exp Brain Res. 2006;172:208-220.

41. Rogers MW, Wardman DL, Lord SR, Fitzpatrick RC. Passive tactile sensory input improves stability during standing. Exp Brain Res. 2001;136:514-522.

42. Ruget H, Blouin J, Coyle T, Mouchnino L. Modulation of proprioceptive inflow when initiating a step influences postural adjustments. Exp Brain Res. 2009.

43. Bloem BR, Allum JH, Carpenter MG, Verschuuren JJ, Honegger F. Triggering of balance corrections and compensatory strategies in a patient with total leg proprioceptive loss. Experimental brain research Experimentelle Hirnforschung. 2002;142:91-107. 
44. Nashner LM, Black FO, Wall C, 3rd. Adaptation to altered support and visual conditions during stance: patients with vestibular deficits. J Neurosci. 1982;2:536-544.

45. Bonnet C, Carello C, Turvey MT. Diabetes and postural stability: review and hypotheses. J Mot Behav. 2009;41:172-190.

46. Freitas SM, Prado JM, Duarte M. The use of a safety harness does not affect body sway during quiet standing. Clin Biomech (Bristol, Avon). 2005;20:336-339.

47. Wu G. Real-time feedback of body center of gravity for postural training of elderly patients with peripheral neuropathy. IEEE Trans Rehabil Eng. 1997;5:399-402.

48. Richardson JK, Sandman D, Vela S. A focused exercise regimen improves clinical measures of balance in patients with peripheral neuropathy. Arch Phys Med Rehabil. 2001;82:205-209.

49. Tessier D, Menard J, Fulop T, et al. Effects of aerobic physical exercise in the elderly with type 2 diabetes mellitus. Archives of gerontology and geriatrics. 2000;31:121-132.

50. van Schie CH. Neuropathy: mobility and quality of life. Diabetes/metabolism research and reviews. 2008;24 Suppl 1:S45-51.

51. Wall C, 3rd, Kentala E. Control of sway using vibrotactile feedback of body tilt in patients with moderate and severe postural control deficits. J Vestib Res. 2005;15:313-325. 52. Dozza M, Wall C, 3rd, Peterka RJ, Chiari L, Horak FB. Effects of practicing tandem gait with and without vibrotactile biofeedback in subjects with unilateral vestibular loss. $J$ Vestib Res. 2007;17:195-204. 


\section{Figure legends}

Fig. 1. Schematic representation of the experimental set-up with a subject positioning on the platform of the Computerized Dynamic Posturography system. A- regular stance without

sensory cues; B- Stance with the device providing sensory cues to the calf area. 1- shank of the brace, 2- foot bed, 3 - semi-rigid element, 4- Velcro calf straps.

Fig. 2. The average SOT scores for four balance tests performed without a device providing sensory cues and with the device. Each bar represents the group mean \pm SE.

Fig. 3. The composite latency scores, ms for the two experimental conditions obtained for nine subjects. Each bar represents the group mean $\pm \mathrm{SE}$.

Table 1. Demographic data 


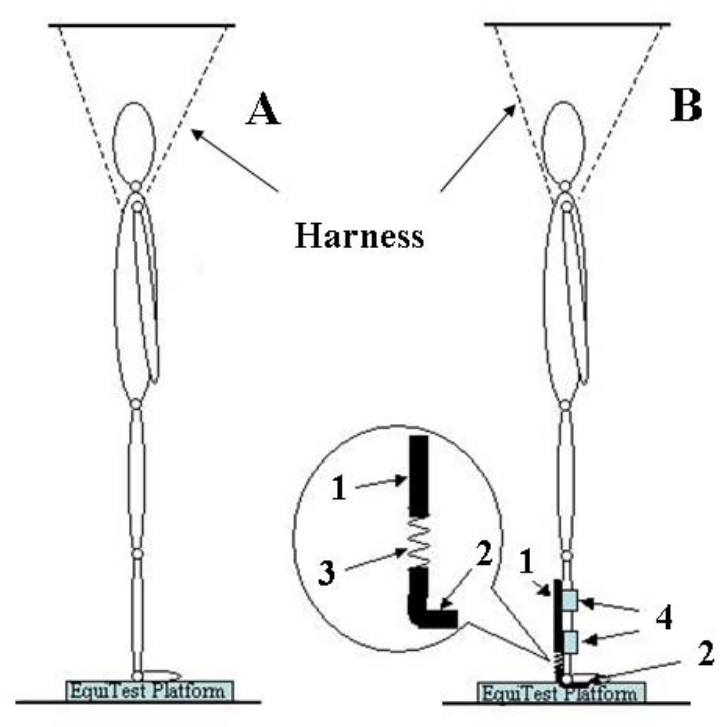

Fig. 1 


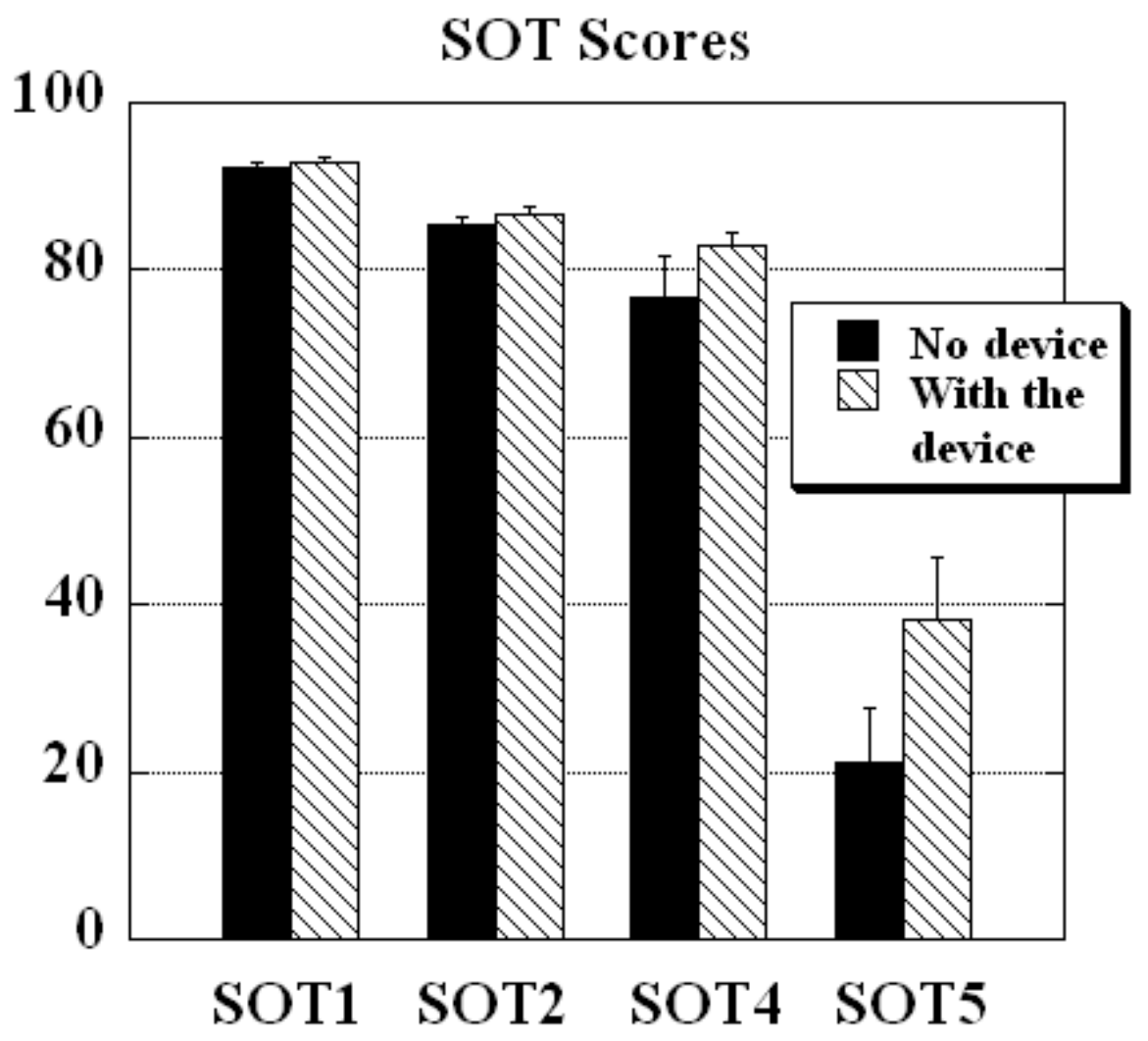

Fig. 2 


\section{Latency Scores}

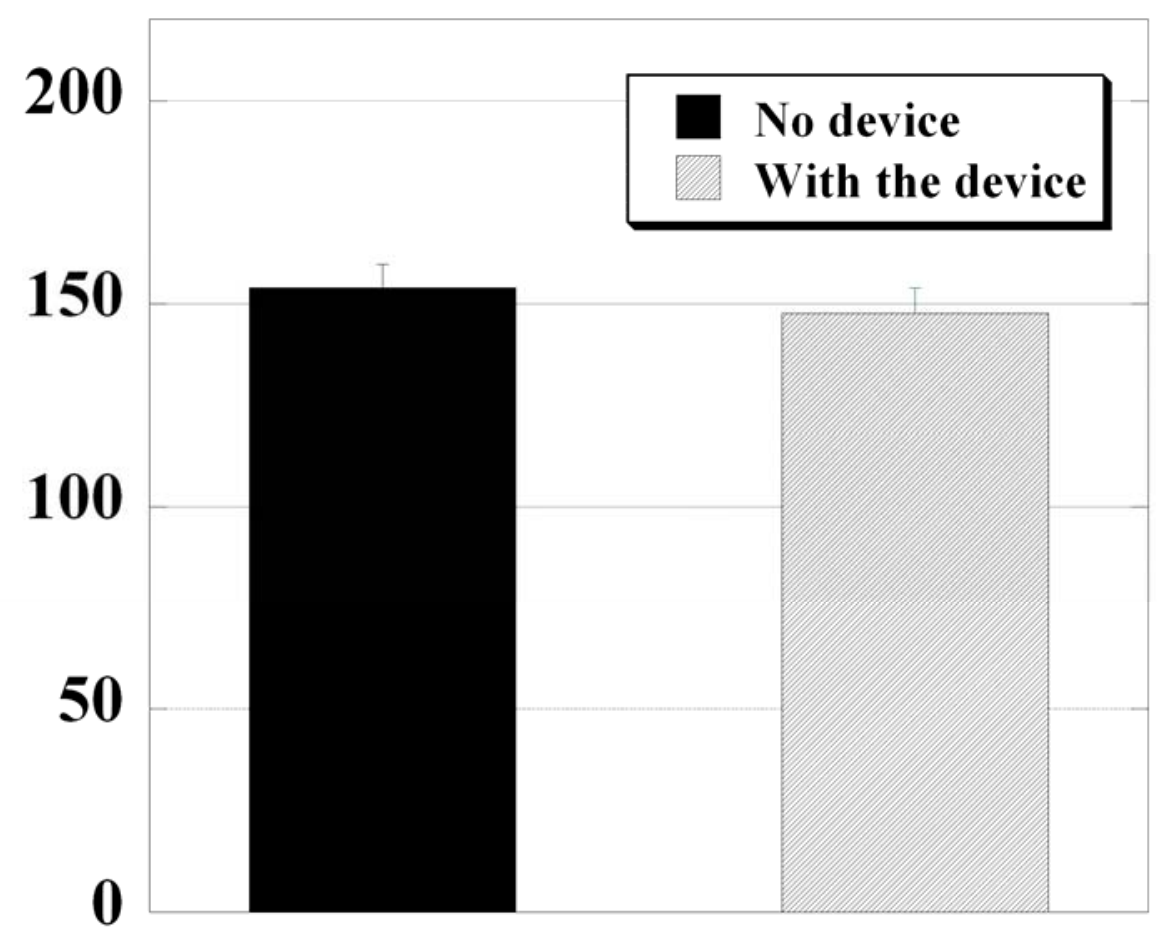

Fig. 3 